\title{
The Effectiveness of Lugol for Endometritis Therapy in Aceh Cow
} \author{
Panjaitan $^{3 *}$ \\ ${ }^{1}$ Laboratory of Reproduction Faculty of Veterinary Medicine Universitas Syiah Kuala \\ ${ }^{2}$ Veterinarian Study Program Faculty of Veterinary Medicine Universitas Syiah Kuala \\ ${ }^{3}$ Laboratory of Clinical Faculty of Veterinary Medicine Universitas Syiah Kuala \\ ${ }^{4}$ Laboratory of Parasitology Faculty of Veterinary Medicine Universitas Syiah Kuala
}

Juli Melia ${ }^{1}$, Baroli Sadri ${ }^{2}$, Tongku N. Siregar ${ }^{1}$, Ginta Riady ${ }^{1}$, Nuzul Asmilia ${ }^{3}$, Muhammad Hanafiah ${ }^{4}$, and Budianto

\begin{abstract}
Endometritis is an inflammation of the uterine wall that may decrease fertility in the short term and may lead to sterility in the long term. This study was conducted to examine the effectiveness of lugol for endometritis therapy in aceh cow. This study used three aceh cows that had been diagnosed endometritis by ultrasonography. The three aceh cows were then treated with $20 \mathrm{ml}$ of $2 \%$ lugol intra-uterine. Observation of the uterine healing process was performed by using transrectal ultrasonography for 24 days after treated with lugol. Parameters observed were uterine diameter, the thickness of the endometrium and the presence of mucus and discharge in the uterus. The result showed that the three cows did not show a significant change. A cream-yellowish discharge came out from the uterus continuously, the diameter of uterine was still large and the sonograph of endometrial displayed as a hyperechoic until the 24th day after therapy. In conclusion, the use of $2 \%$ Lugol as a therapy for endometritis in aceh cows during 24 days is not effective on the uterine healing of endometritis's cows.
\end{abstract}

Keywords: aceh cow, lugol, endometritis and ultrasonography.

\section{Introduction}

The efforts to increase the number of local cattle such as Aceh cattle is one of the important strategies for the fulfillment of the meat requirements in Indonesia.The lack of cow maintenance management such as feeding management, maintenance environment, weaning and prevention of disease are the causes of reproductive disorders [1]. One of the reproductive disorders is endometritis.

Endometritis is an inflammation of the uterine mucosa (endometrium). This disease can be temporary (infertile) or permanent (advanced) [2]. The prolonged uterine infection results in decreased reproductive capacity caused of the abnormal of uterus and ovaries function. Ultrasonography can be used to investigate the condition of the uterus with endometritis [3]. Endometritis sonograms are characterized by enlarged uneven uterine surface size and found to be anechoic-hyperechoic fluid in the uterine lumen [4].

Various therapies in cows with endometritis have been carried out using antibiotics, prostaglandins [5,6,7], and Lugol $[8,9,10]$. The use of Lugol $1 \%$ can treat reproductive disorders of cattle like uterus infection [11]. The use of Lugol 2\% has a good effect on buffaloes undergoing anesthesia [12]. The use of Lugol as an alternative therapy for endometritis in local cows especially aceh cows through uterine sonography observations is still absent. Based on these reasons, this study was conducted to determine the effectiveness of Logol for endometritis treatment through a observation of changes in the uterus using ultrasonographic (USG). Therefore, it is expected to provide more accurate and clear information about the best course of action for endometritis therapy in aceh cow.

\section{Materials and Methods}

This study used three aceh cows that had been diagnosed with endometritis SA1, SA2, and SA3, aged 5 to10 years old, body weight $150-250 \mathrm{~kg}$. The diagnosis of endometritis inaceh cows was performed based on the clinical examination using an ultrasonography (Mindray DP 10 vet with 50L60EAV linear transrectal probe Shenzhen Mindray Bio-Medical Electronics Co., Ltd.) and the Metricheck (Simcro, New Zealand). Cows that have endometritis are treated using $20 \mathrm{ml}$ of Lugol 2\% intra uteri. Observation of the uterine healing process was carried out using transrectal ultrasonography based on uterine diameter, the thickness of the endometrium and the presence of pus and mucus in the uterus. The observation was carried out before and after therapy using lugol for 24 days.

\subsection{Clinical Examination}

Clinical examination on the reproductive organs of female aceh cow is carried out by observing the presence of fluid

* Corresponding author: antopjt@gmail.com 
in the uterus and also the discharge around the vulva and tail.

\subsection{Ultrasonography}

Cows were are put into the nostal, ultrasonography was placed in a safe place from the cow and is easily accessible by the operator. The surface of the transducer was coated with an ultrasound Gel (Cosmo Med®) and coated with thin plastic then the transducer is inserted into the rectum along the tractus [13].

The observed parameters were the diameter of the uterus (cervix, corpus and cornua uteri) measured transversely from the longest axis. Endometrial thickness was measured sagittally in the uterine wall bounded by the lumen and the presence of pus and mucus using the sonogram.

\subsection{Data Analysis}

The data obtained in this study were analyzed descriptively based on the images (graphs and sonograms) obtained from USG.

\section{Results and Discussion}

\subsection{Clinical Examination}

The clinical appearance of Aceh cows with endometritis showed the presence of residual discharge around the tail, vulva, and perineum (Figure 1). The examination of endometritis discharge using Metricheck showed a score of 3-4. In addition, the diagnosis was also conducted by rectal palpation. Enlargement of the uterus indicated that the uterus was abnormal. Transrectal palpation is a diagnostic method that can be used to see the uterus return to normal, but it is difficult to identify because can be a subjective result [14].

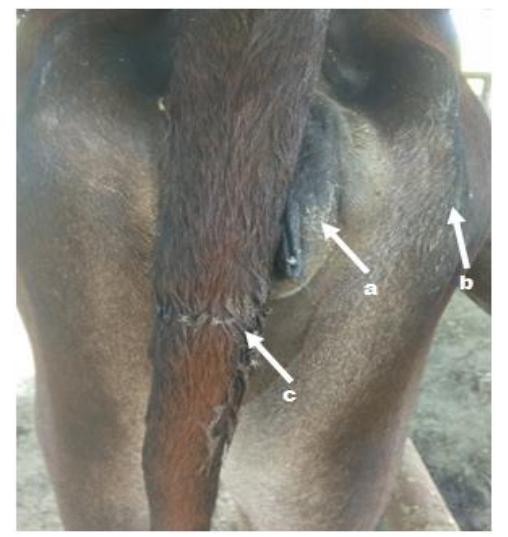

Figure 1. Clinical symptoms in Aceh cows with endometritis. Discharge appears attached to the vulva (a), perineum (b), and tail (c).

\subsection{The presence of mucus and pus in the uterus}

Uterine sonography with endometritis shows hyperechoic features of the uterine lumen [3]. This is consistent with the results of research on aceh cows that were diagnosed with endometritis. Uterine sonography shows abnormal hypoechoic to hyperechoic mucus in the lumen (Figure 2).

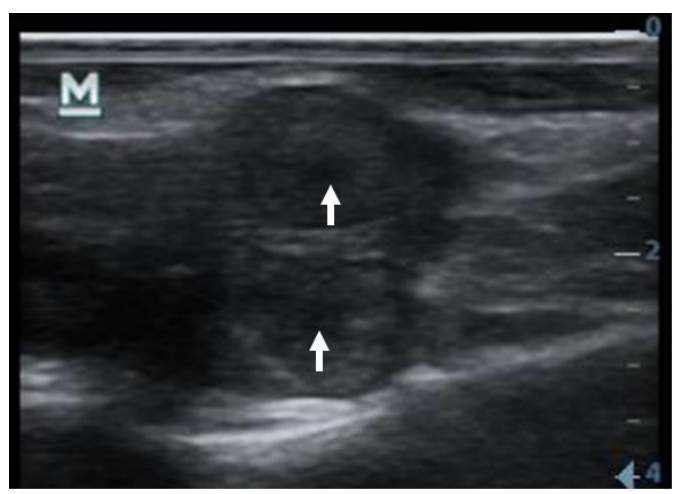

Figure 2. Mucus ultrasonographic features of the uterine cornua. Arrows $=$ discharge.

After performing Lugol therapy in three endometritis cows, the appearance of brownish discharge discharged up to 3 days after being treated. On SA1 the discharge turns yellow from day 4 (D4) to day 24 (D24). The color changed to yellow also occurs in SA2 which is seen since D4-D19, then changes to beige on D20-D24, while in SA3 the mucus changed from brown to yellow from D11-D17 and turn to beige on D20-D21. After that, no discharge was found. The appearance of abnormal mucus after $2 \%$ Lugol therapy in aceh cows with endometritis is presentedin Figure 3.

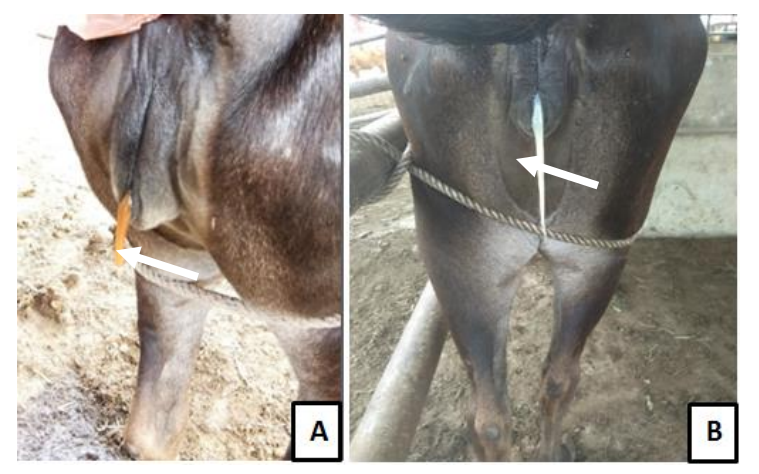

Figure 3. The appearance of abnormal mucus that comes out of the cow's vulva endometritis after treatment of lugol $2 \%$. A. One day after therapy (D1); B. Four days after therapy (D4). Arrows $=$ discharge.

Mucus removal is an attempt by the body to do homeostasis so that the body can return to normal. The mechanism of immunity of the uterus to disorders of the reproductive system can be done anatomically and chemically, such as mucus secretion from the endometrium [15]. The use of lugol can affect the contraction of the myometrium and increase blood supply from the uterus [16]. In Aceh cows, this contraction can be seen from the reaction of cows which constantly press after Lugol administration. Along with the journey, the discharge comes out.

\subsection{Uterine Diameter}

Based on observations before therapy, cervical SA1 data had a size of $1.88 \mathrm{~cm}$, after therapy an increase in cervical diameter to $1.90 \mathrm{~cm}$ in D1 then decreased in D2-D3 (1.67 
$\mathrm{cm})$ and again increased in D4 $(2,05 \mathrm{~cm})$ then the cervix continues to decrease in size to D10 $(1.26 \mathrm{~cm})$, while the cervical D11-D24 undergoes fluctuating changes. Changes also occur in the diameter of the corpus and cornua of the uterus. The diameter of the corpus before being treated (D0) has a size of $1.46 \mathrm{~cm}$, the day after being treated (D1) an increase in size becomes $1.77 \mathrm{~cm}$ and continues to experience fluctuating changes up to D3.
Decreased corpus size occurs at D4-D10 then returns to fluctuating size changes to D24. Before therapy, the cornua was $1.04 \mathrm{~cm}$ in size, then increased in diameter at D1-D5 to $1.39 \mathrm{~cm}$ in size than on D6-D8 it decreased to $0.9 \mathrm{~cm}$ and the change in size continued until D24. Changes in diameter on SA1 can be seen in Figure 4.

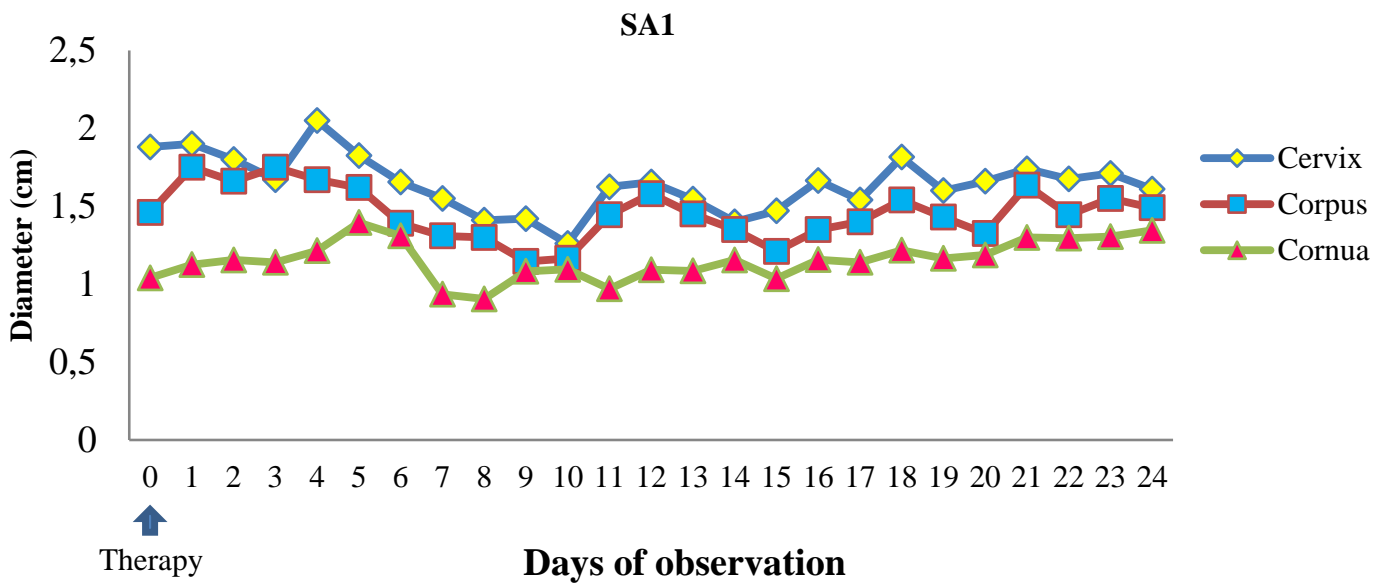

Figure 4. Changes in diameter of the cervix, corpus, and cornua uterus SA1 (Aceh 1 cow) treated with Lugol $2 \%$.

The size of the cervical diameter SA2 before therapy $(2.03 \mathrm{~cm})$ subsequently on D1 increased $(2.55 \mathrm{~cm})$ then D2 decreased in size to D7 $(2.04 \mathrm{~cm})$ and changes in cervical size continued to occur flexibly until D24. Before being treated the corpus has a size $(1.99 \mathrm{~cm})$ after therapy (D1) until D3 has increased $(2.24 \mathrm{~cm})$ then D4-D5 shows a decrease to $1.52 \mathrm{~cm}$ and the size of the corpus continues to experience fluctuating changes until D24. The size of the cornua SA2 before therapy $(1.16 \mathrm{~cm})$ and then after therapy (D1) increased to $1.23 \mathrm{~cm}$ and changes in size that occurred fluctuatively continued until D24 observation. Changes in the diameter of the SA2 uterus can be seen in Figure 5.

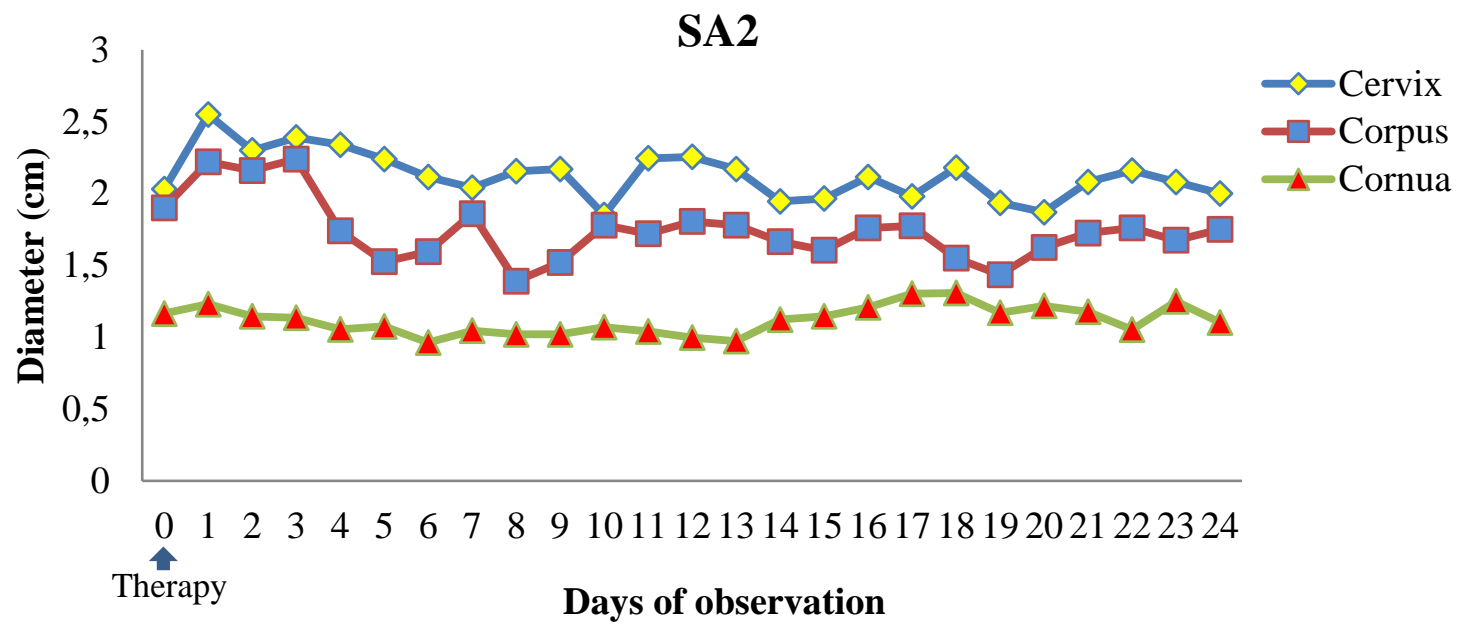

Figure 5. Changes in diameter of the cervix, corpus, and cornua uterus SA2 (Aceh 2 cow) treated with Lugol $2 \%$.

Before therapy on cervical diameter SA 3 is $2.02 \mathrm{~cm}$, after cervical D1-D3 therapy has increased $(2.15 \mathrm{~cm})$, then decreased to $1.98 \mathrm{~cm}$ on $\mathrm{D} 4$, then cervical diameter continues to experience fluctuating changes until D12 ( $2.1 \mathrm{~cm})$ and decreased again from D13-D14 to reach the size of $1.55 \mathrm{~cm}$ and increased again at D15-D19 $(2.30 \mathrm{~cm})$ on the following day decreased to D24 with a diameter of
$1.85 \mathrm{~cm}$. Before therapy, the corpus has a diameter of 1.84 $\mathrm{cm}$ then an increase after being treated up to D3 $(2.01 \mathrm{~cm})$ on $\mathrm{D} 4$ decreased in size to $1.74 \mathrm{~cm}$ and the next day there was a fluctuating change in corpus size up to D24. Cornua SA3 at D0 has a size of $0.85 \mathrm{~cm}$ and changes fluctuatively to D24 $(1.11 \mathrm{~cm})$. Changes in SA3 diameter are presented in Figure 6. 


\section{SA3}

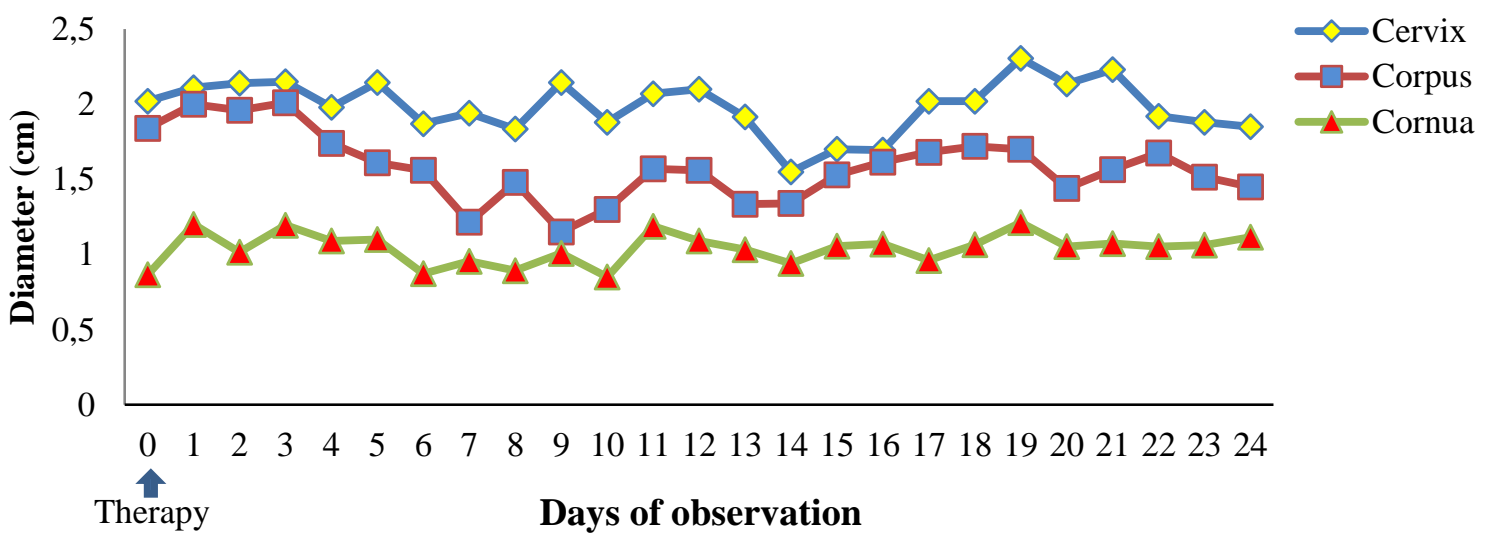

Figure 6. Changes in diameter of the cervix, corpus, and cornua uterus SA3 (Aceh 3 cow) treated with lugol $2 \%$.

Increasing cervical diameter after lugol treatment is in line with discharge, but changes in uterine diameter that occur fluctuatively indicate that lugol administration has not been effective for endometritis therapy in Aceh cows. Uterine sonography shows changes in diameter after the treatment of lugol in Aceh cows endometritis (Figure 7). The use of lugol can cause contraction of the myometrium and increase blood supply to the uterus [16]. But this study has not shown a smaller diameter of the uterus compared to before it was treated until D24.
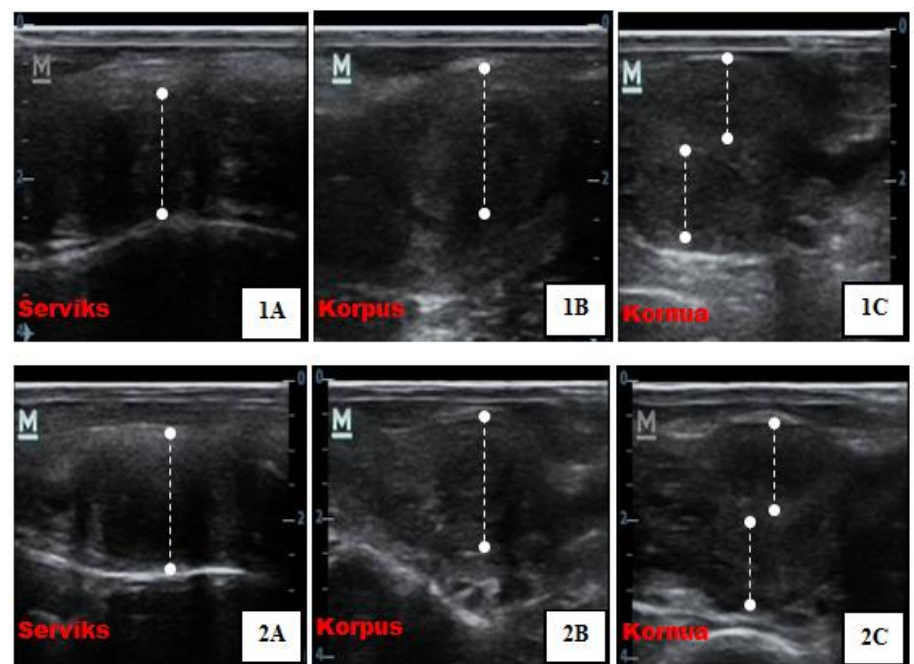

Figure 7. Ultrasound features of cervical, corpus and cornua uterus of bovine Aceh endometritis. Before (1A, B, C) and after treatment with lugol $2 \%(2 \mathrm{~A}, \mathrm{~B}, \mathrm{C})$.

\subsection{Endometrial thickness}

Cattle that have endometritis generally have endometrial walls thicker caused by the formation of connective tissue in the uterus in response to the immune system of the uterus. The results showed that SA1 before being treated with lugol had an endometrial thickness of $0.60 \mathrm{~cm}, \mathrm{D} 1-$ D2 had increased to $0.99 \mathrm{~cm}$ and D3 had decreased endometrium thickness fluctuating up to D24.
The thickness of the endometrium SA2 D0 is $0.64 \mathrm{~cm}$ then an increase in D1-D3 to $1.00 \mathrm{~cm}$ and D4 slowly begins to experience a fluctuating decrease in the size of the endometrium to D24. The thickness of the endometrium SA3 before therapy is $0.54 \mathrm{~cm}$ after being treated with D1-D4, increasing the thickness of the endometrium to $0.88 \mathrm{~cm}$ and D5-D24, the thickness of the endometrium decreases fluctuatively (Figure 8). 


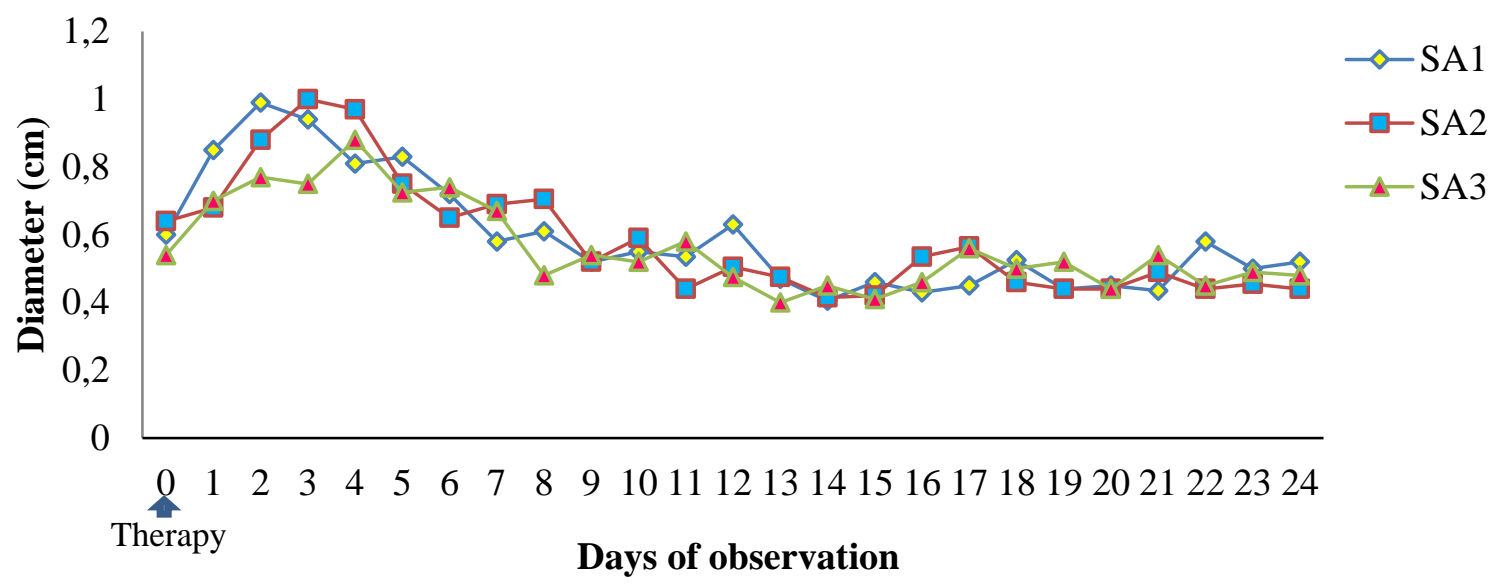

Figure 8. Endometrial wall thickness in Aceh endometritis cows treated with lugol $2 \%$.

Endometrial wall thickness in Aceh endometritis cows treated with lugol 2\%. Endometrial sonography before treatment shows hyperechoic features that showing the presence of connective tissue in the endometrium. After treatment using endometrial lugol sonography, hyperechoic features still appear (Figure 9). Based on the observation of endometrial thickness after lugol therapy in Aceh cattle endometritis has not shown a good effect. Intrauterine use of antiseptics as a therapy for reproductive disorders has not been effective in reproductive performance [5].
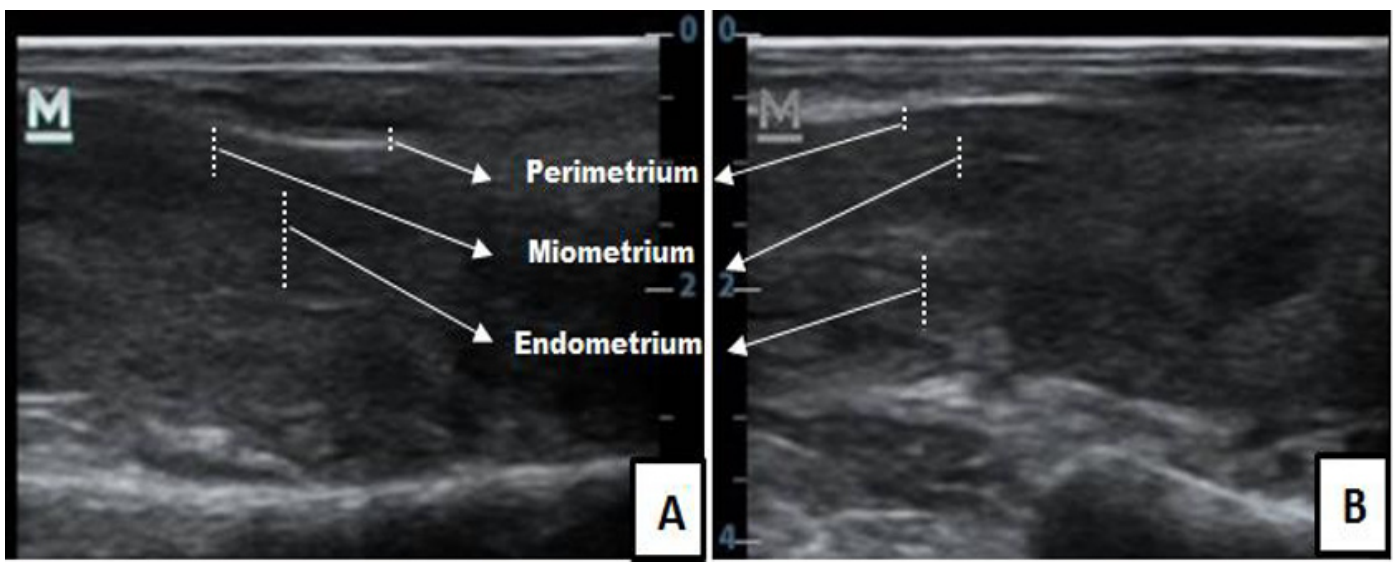

Figure 9. Ultrasound picture of the endometrial lining of bovine Aceh endometritis patients before (A) and after (B) $2 \%$ lugol therapy.

Therapy has given in the form of antiseptic one of which is lugol [17]. The use of lugol $1 \%$ can treat reproductive disorders in cattle [8.11]. Therapy using $2 \%$ lugol in buffalo can have a good effect on reproductive disorders and $2 \%$ lugol in buffalo can have a good effect on reproductive disorders [12]. But the use of lugol with a concentration of $2 \%$ in this study as a therapy for Aceh cattle endometritis has not been able to give a good effect. This may be because the concentration of lugol used as a therapy is too concentrated. The use of iodine as a therapy for reproductive disorders has irritating side effects and causes necrose in the uterine mucosa [18]. Treatment of reproductive disorders using lugol causes transient acute inflammatory reactions in the endometrium but does not cause permanent endometrial fibrosis. It is likely that this also occurs in Aceh cattle with endometritis after lugol treatment of $2 \%$ [18].

\section{Conclusion}

The $2 \%$ lugol therapy has not been able to give a good effect on the healing process of endometritis in aceh cows based on the appearance of uterine ultrasonography.

\section{References}

1. Sutiyono, Samsudewa D, Suryawijaya A: Identifikasi gangguan reproduksi sapi betina di peternakan rakyat. J. Veteriner. 2017; 18(4): 580-588.

2. Melia J, Amrozi, Tumbelaka LI, et al.: Identification of Polymorphonuclear (PMN) Leucocytes in Blood of Endometritis Cows Treated with a Combination of Intrauterine Infusion of Gentamicine, Flumequine, and PGF2a Analog Flumequin, dan analog PGF2a. J. Ked. Hewan. 2012; 6(2): 117-119.

3. Descoteaux L, Gnemmi G, Colloton J: Practical Atlas Of Ruminant And Camelid Reproductive Ultrasonography. Wiley-Blackwell. USA. 2010. 
4. Noviana D, Aliambar SH, Ulum MF, et al.: Diagnosis Ultrasonografi pada Hewan Kecil. Ed.II. IPB Press. Bogor. 2018.

5. Drillich M: An update on uterine infections in dairy cattle. Slov. Vet. Res. 2006; 43(1): 11-5.

6. Mido S, Murata N, Rawy MS, et al.: Effects of intrauterine infusion of povidone-iodine on endometrial cytology and bacteriology in dairy cows with clinical endometritis. J. Vet. Med. Sci. 2016; 78(4): 551-556.

7. Szenci O: Recent possibilities for diagnosis and treatment of post parturient uterine diseases in dairy cow. JFIV Reprod. Med. Genet. 2016; 4(1): 1-7

8. Ahmed FA, Saxena M, Ravikanth K, et al.: A herbal intrauterine infusion "Uraksha Liquid" for treatment of reproductive disorders in cows. IJPRBS. 2014; 3(2): 42-48.

9. Ahmed FO, Elsheikh AS: Treatment of repeat breeding in dairy cows with Lugol's Iodine. IOSR-JAVS. 2014; 7(4): 22-26.

10. Alyasiri EA, Alwan AF, Al-Hamedawi TM: Comparative study of some intrauterine treatment regimes on bacterial causes of repeat breeders in Iraqi buffaloes. Paripex - Indian Journal of Research. 2015; 4(9): 188-190.

11. Ahmed FO, Elsheikh AS.: Intrauterine infusion of lugol's iodine improves the reproductive trais of postpartum infected dairy cows. IOSR-JAVS. 2013; 5(2): 89-94.
12. Ahlawat AR, Gajbhiye PU, Odedra MD, et al.: Effect of lugol's iodine on estrus induction and fertility response in true anestrus jaffrabadi buffaloes. Buffalo Bulletin. 2016; 35(3): 303-305.

13. Melia J, Amrozi, Tumbelaka LI: Ovarian Dinamics of Endometritis Cows Treated with a Combination of Intrauterine Infusion of Gentamicine, Flumequine, and PGF2a Analog. J. Ked. Hewan. 2014; 8(2): 111-115.

14. Dolezel R, Vecera M, Palenik T, et al.: Systematic clinical examination of early postpartum cows and treatment of puerperal metritis did not have any beneficial effect on subsequent reproductive performance. Veterinarni Medicina. 2008; 53(2): 59-69.

15. Dhaliwal GS, Murray RD, Woldehiwet Z: Some aspects of immunology of the bovine uterusrelated to treatments for endometritis. Animal Reproduction Science. 2001; 67(1): 135-152.

16. Hussain SO, Al-Zubaidi SF dan Asofi M.: Different endometritis treatments in ewe: comparative study. IOSR-JAVS. 2013: 3(5): 91-94.

17. Smith BI, Risco CA: Clinical manifestation of postpartum metritis in dairy cattle. Comp Contin Educ Pract Vet. 2002: 24: S56-S63.

18. Nakao T, Moriyoshi M, Kawata K: Effect of postpartum intrauterine treatment with $2 \%$ polyvinylpyrrolidone- iodine solution on reproductive efficiency in cows. Theriogenology. 1988; (30): 1033-1043. 